\title{
LA ESTRUCTURA RIZOMÁTICA DE LA INCIDENCIA 2.0
}

\section{THE RHIZOMATIC STRUCTURE OF ADVOCACY 2.0}

\section{VERÓNICA YÉPEZ-REYES ${ }^{1}$}

Recibido: 4 de enero de 2017

Aceptado: 6 de marzo de 2017

\footnotetext{
${ }_{1}^{1}$ Pontificia Universidad Católica del Ecuador, Facultad de Comunicación, Lingüística y Literatura, Escuela de Comunicación, Quito, Ecuador (vyepezr@puce.edu.ec).
} 



\title{
LA ESTRUCTURA RIZOMÁTICA DE LA INCIDENCIA 2.0
}

\section{THE RHIZOMATIC STRUCTURE OF ADVOCACY 2.0}

\author{
VERÓNICA YÉPEZ-REYES
}

\begin{abstract}
PALABRAS CLAVES: metáfora del rizoma, interacción digital, Incidencia 2.0, comunicación para el cambio social, justicia global
\end{abstract}

KEYWORDS: rhizome metaphor, digital interaction, Advocacy 2.0, communication for social change, global fairness

\section{RESUMEN}

Este artículo se basa en la metáfora del rizoma propuesta por Gilles Deleuze y Felix Guattari para analizar la estructura que mejor describe a la interacción digital promovida por las redes de organizaciones de movimientos por la justicia global. Un tema central en este análisis es la comprensión de la comunicación para el cambio social sustentada en la interacción digital, definida como el proceso de comunicación de múltiples vías mediadas por la Internet y el uso de las TICS. A lo largo de dieciocho meses se siguió la interacción digital en las cuentas de Twitter y Facebook de cinco organizaciones europeas y sus ramas locales para el Ecuador. El análisis propone que la estructura de la interacción digital denominada Incidencia 2.0, en parangón con el desarrollo de la Internet y la web 2.0, está provista de una estructura rizomática con múltiples, diversas y dispersas temáticas, preocupaciones, redes e idiomas aparentemente desconectados pero que en la práctica están entrelazados. Queda abierto el campo de investigación para el análisis acerca de cómo esta estructura facilita para que los contenidos se transformen en imaginarios sociales y a su vez en herramientas para el cambio social. 


\section{ABSTRACT}

This article builds on Gilles Deleuze and Felix Guattari rhizome metaphor, for the analysis of the structure that best describes digital interaction promoted by global fairness organizations. A key issue in the analysis is the understanding of communication for social change sustained on digital interaction, which is defined as the multi-ways communication process mediated by the Internet and the use of ICTs. During eighteen months the study followed digital interaction taking place in the Twitter and Facebook accounts of five European organizations and their local branches for Ecuador. The article suggests that digital interaction, termed as Advocacy 2.0 -in straight relation with the development of the internet and the web 2.0-is provided by a rhizomatic structure with multiple, diverse and disperse matters, concerns, networks and languages that are apparently disconnected but interrelated in practice. The research field remains open for the analysis of how this structure could provide the means for the contents in digital interaction to transform into social imaginaries and as such, into tools for social change.

\section{INTRODUCCIÓN}

En este artículo la estrecha relación que existe entre las redes de organizaciones de movimientos por la justicia global (Della Porta, 2009b; Steger et al., 2013) y la comunicación para el cambio social (Nos Aldás \& Pinazo, 2013; Tufte, 2013, 2015; Wilkins, 2014) toma como punto de partida los eventos ocurridos en Seattle en 1999, en donde un cúmulo de movimientos sociales "se involucraron en movilizaciones disruptivas que presagiaron la formación de un movimiento social transnacional focalizado en la globalización" (Zárate, 2015: p. 70).
Si bien a fines de la década del 90 ya existía el activismo conectado entre organizaciones de base, organizaciones no gubernamentales (ONGs) locales y ONGs internacionales encaminado al cabildeo, la defensa e influencia. En 1998, Margaret Keck y Kathryn Sikkink acuñaron el acrónimo TANs (por su siglas en inglés: transnational advocacy networks) para referirse a las redes transnacionales de incidencia encargadas de posicionar en la arena internacional conflictos locales, apoyar a los activistas y a las luchas locales del sur y del norte 
global y facilitar encuentros internacionales que fortalecerían las redes creadas por las tecnologías de información y comunicación (TICs) de la época: fax, teléfono y correo convencional y electrónico. Las TANs toman mayor impulso a partir de la conformación del movimiento por la justicia global a partir del año 2000.

No existe consenso entre autores para referirse a "un movimiento" o a una red de movimientos por la justicia global (Della Porta, 2009a). El desacuerdo surge de la contradicción que supone unificar la diversidad propia de este movimiento, mediante universales centralizados que niegan la complejidad de intereses e identidades confrontados en los procesos de globalización.

Por ello, estos han sido referidos como "movimientos sociales rizomáticos" en los estudios de Manuel Castells (2012), Arturo Escobar y Michal Osterweil (2010) o Peter Funke (2012). La metáfora del rizoma propuesta por Gilles Deleuze y Feliz Guattari (1987) se convierte en una herramienta útil para el análisis de la complejidad de estos movimientos sin un eje central definido.

En 2006, Lance Bennett propone diferenciar dos eras en el estudio del activismo transnacional: la era de las TANs y la era del/los movimiento/s por la justicia global. Estas dos eras también pueden analizarse en parangón con el desarrollo de la Internet, denominando a la era de las TANs como Incidencia 1.0.

La Incidencia 1.0 corresponde a una red típicamente organizada de forma centralizada a partir de una sede medular que lidera los procesos de incidencia, encargada además de establecer la agenda de trabajo e intermediar el contacto entre coaliciones de organizaciones. De igual manera, la web 1.0 se identifica por ser una red de comunicación de un solo sentido, en donde un administrador genera la información que se transmite a los usuarios (Yépez-Reyes \& Dohn, 2016).

La Incidencia 2.0 en cambio se fundamenta en la web 2.0, en donde la comunicación es generada por los propios usuarios y mediada por la red en un proceso de comunicación de múltiples vías (Dohn \& Johnsen, 2009). Está caracterizada por la interacción digital, definida como los procesos de comunicación de múltiples vías mediados por la Internet y el uso de las TICs.

En la Incidencia 2.0 toma fuerza el movimiento por la justicia global también llamado "el movimiento de movimientos" (Della Porta \& Mosca, 2005; Willetts, 2011) que reúne a una amplia variedad de movimientos sociales: de género, derechos humanos, educación, ambiente, alimentación, por la paz, para nombrar unos pocos, los cuales desde su propio ámbito, desafían el estatus 
hegemónico de la ideología dominante de la globalización de mercado (Steger et al., 2013). De igual manera, al internet también se lo define como una "red de redes" que genera esta gran autopista de información. En la Incidencia 2.0, las organizaciones han tenido que adaptarse a las nuevas condiciones y adoptar nuevos e innovadores mecanismos de acción (véase por ej. Ackland \& O’Neil, 2013; Córdoba \& Jansen, 2015). Por ello, Bennett y Alexandra Segerberg proponen en 2012 que la lógica de la interacción digital define tres tipos de redes:

- de acción colectiva intermediada por organizaciones,

- de acción conectiva facilitada por organizaciones y

- de acción conectiva facilitada por las multitudes.

Nótese la diferenciación entre acción colectiva y acción conectiva; la primera referida al tipo de acción que puede o no tener lugar con el apoyo de las TICs, mientras los otros dos tipos de acción son facilitados a través de la Internet y el uso de las TICs.

Actualmente existe un número creciente de literatura enfocada a analizar los movimientos sociales del siglo XXI que se caracterizan por la acción de multitudes, descentralizadas y autoconvocadas a través de la Internet y las redes sociales, tales como la Primavera Árabe, Ocupa Wall Street o los Indignados del 15-M en España (Castells, 2012; Christensen, 2011; García-Alabacete \& Theocharis, 2014; Toret, 2013). Sin embargo, este capítulo comparte la percepción de Bennett y Segerberg (2013: p. 48) que la acción conectiva facilitada por las organizaciones es el "tipo más intrigante de los tres", por ser un híbrido entre los otros dos.

El concepto de "affordances" planteado por James Gibson (1986) es útil para describir como la Internet y el uso de las TICs facilita la Incidencia 2.0. Este concepto que describe las potencialidades de uso de un objeto, ha sido aplicado exitosamente al estudio de la interacción humano-computador - $\mathrm{HCl}$, por sus siglas en inglés: human computer interaction- (Conole, 2013; Dohn, 2009; Lauridsen \& Hansen, 2016). De acuerdo con Gibson:

- Las affordances son objetivas en tanto son independientes de la persona que utiliza el artefacto. Cada objeto tiene al menos un affordance, a pesar que el usuario no emplee el artefacto de esa manera; por ejemplo, una silla es un artefacto diseñado para sentarse, aunque yo pudiese pararme sobre ella para alcanzar el tumbado, o podría llenarla de ropa, maletas y carteras ocultándola completamente. 
- Las affordances son subjetivas ya que están también determinadas por el usuario y por ende dependen "de la cultura, las experiencias previas y la educación" (Dohn, 2009: p. 155). En el ejemplo anterior, utilizar la silla como si fuese una escalera o un vestidor, son dos affordances subjetivas para un mismo objeto diseñado como asiento.

Jennifer Earl y Katrina Kimport (2011) mencionan dos affordances claves de la Internet para el activismo conectado:

- Costos reducidos para la organización y participación en acciones, gracias a la reducción de presupuestos en llamadas, envíos, faxes e impresiones y en reuniones de movilización para la planificación de acciones y eventos.

- Disminución de la necesidad de presencia física, cara a cara, para la acción colectiva, Castells (2012) sostiene que esto permite que se generen redes híbridas, locales y globales, individualizadas y conectadas, presentes en el ciberespacio y ocupando espacios físicos en el tiempo atemporal de la red.

La interacción digital abierta a la participación es fundamental para la Incidencia 2.0. Por tanto, una tercera affordance la constituyen las redes sociales, multiplicando los destinatarios de un tema por sobre los límites físicos de una conversación presencial. Más aún, Anastasia Kavada (2012) sugiere que las publicaciones en redes sociales se vigorizan gracias a un sentido de unidad, pertenencia y confianza provisto por la redes virtuales y físicas a la vez.

La Incidencia 2.0 se inserta dentro del campo de la comunicación para el cambio social el cual Thomas Tufte (2013) sugiere que surgió en América Latina, como una corriente sustentada en la práctica de la Pedagogía de la Liberación de Paulo Freire (1985). Así, la comunicación para el cambio social busca generar procesos de comunicación participativa, enmarcados en una "praxis" en el sentido dialógico freiriano de reflexión-acción.

\section{TWITTER Y FACEBOOK PARA LA INTERACCIÓN DIGITAL}

Tim O'Reilly (2007), uno de los fundadores del concepto de la web 2.0, describe a las redes sociales como las aplicaciones en internet que han sacado 
el mayor provecho de las ventajas intrínsecas de esta plataforma, constituyéndose en canales de comunicación hechos a la medida de los usuarios. Clay Shirky (2008) añade que las redes sociales cumplen un rol importante tanto como nuevos medios de comunicación para publicar y compartir acciones coordinadas como para construir colaborativamente conocimientos

Twitter empezó en 2006 como un servicio de mensajería de textos observando el límite de 140 caracteres establecido para la telefonía móvil. Este límite se mantiene hasta la actualidad, pero hoy las publicaciones (tweets) pueden incluir imágenes, audio y video. Twitter es una plataforma de microblogging que cada día registra aprox. 500 millones de tweets en sus 35 idiomas'; Twitter registró en el segundo trimestre de 2015 más de 316 millones de usuarios activos mensuales².

La estructura de Twitter es muy simple, se compone de tweets, re-tweets (reenvíos), menciones a otras cuentas utilizandola @ precediendo al nombre de la cuenta, \# hashtags precediendo a temas que constituyen enlaces cortos a términos destacados y vínculos a pá- ginas de internet usualmente incluidos mediante acortadores de direcciones (por ej. bit.ly o goo.gl). En principio todas las cuentas de Twitter son públicas, no obstante sí es posible modificar las condiciones de privacidad de la cuenta para que exista reciprocidad entre usuarios.

Las affordances de Twitter para el análisis cuantitativo de la información están dadas por su información corta, concisa y con fechas y horas fijas. Sin embargo, estas mismas affordances son inconvenientes para el análisis cualitativo de la información principalmente por la ausencia de una narrativa clara y localizada.

Facebook se abrió al público en 2006, concebido como la versión digital del "face book", un directorio impreso de fotografías y nombres que las universidades en los Estados Unidos utilizaban para identificar a los estudiantes en el campus. En el segundo trimestre de 2015, Facebook contó con 1.49 billones $^{3}$ de usuarios activos al mes; maneja 70 idiomas distintos y provee herramientas de traducción para la mayoría de ellos.

Mimi Marinucci (2010) argumenta que el anonimato y la invisibilidad están ausentes en la filosofía de Facebook.

\footnotetext{
${ }^{1}$ Twitter. Twitter Use. https://about.twitter.com/es/company Retrieved 16.09.2015

${ }^{2}$ Twitter. Financial releases. Twitter Q2'15 Earnings Release. July 28, 2015. https://investor.twitterinc.com/ releasedetail.cfm?ReleaselD=924259 Retrieved 24.09.2015

${ }^{3}$ En inglés de los Estados Unidos, un billón es entendido como diez mil millones $\left(10^{10}\right)$, y no como el millón de millones $\left(10^{12}\right)$ de otros idiomas como el español. Facebook. Facebook Reports Second Quarter 2015 Results. July 29, 2015
} 
Lo comentarios en Facebook los escribe un participantes que tiene a su vez una red de conocidos en el mundo físico que amparan su presencia en el mundo virtual. Inicialmente la reciprocidad entre usuarios era necesaria en Facebook, pero a partir de 2011 la plataforma introdujo la posibilidad de suscripción a cuentas para leer actualizaciones públicas.

Facebook tiene tres tipos de cuentas: perfiles personales, páginas y grupos. Las organizaciones, los negocios y las marcas deben manejar una página, aunque una persona con un perfil será responsable de su manejo. Las páginas están abiertas a un número ilimitado de seguidores (restringido a 5000 amigos en perfiles personales) y a estadísticas de manejo y seguimiento del tráfico de información.

Las actualizaciones de estado pueden ser publicadas tanto en perfiles personales propios cuanto en perfiles de la red de amigos. Las publicaciones pueden constar de texto, imágenes, videos y enlaces. Se conectan a través de etiquetas conversacionales o fotográficas que funcionan de la misma manera que las menciones@en Twitter.

El uso del Like (Me gusta) en Facebook, es una característica de este medio ahora también compartida con el ícono del corazón en Twitter. Durante los 18 meses de esta investigación la única reacción posible a una publicación en Facebook fue el Like, no obstante a partir del 24.02.2016 Facebook lanzó las reacciones animadas: Me Gusta, Me Encanta, Me Divierte, Me Asombra, Me Entristece, Me Enoja, que aparecen al pulsar o desplazar el mouse sobre el botón de Like.

\section{MÉTODOS Y MATERIALES}

Este articulo operacionaliza la metáfora del rizoma propuesta por Deleuze y Guattari en la introducción de su obra seminal A Thousand Plateaus: capitalism and schizophrenia (1987) para analizar la interacción digital en la Incidencia 2.0. Esta metáfora se basa en el funcionamiento de las plantas rizomáticas como los manglares o el bambú que han desarrollado a lo largo del tiempo un número de adaptaciones dadas las condiciones dinámicas de su hábitat, por tanto no cuentan con un único centro, no poseen límites definidos lo que les permite dispersarse en múltiples direcciones y de maneras heterogéneas a través de nodos semiindependientes que se renuevan, crecen y regeneran por sí mismos.

La selección de las organizaciones del estudio se basó en un "diseño 
múltiple de casos" (Yin, 2014) que incluye "casos paradigmáticos" (Flyvbjerg, 2006) de redes interconectadas por la justicia global. Las organizaciones seleccionadas cumplen con dos criterios básicos:

a) organizaciones de origen europeo con alcance transnacional y trabajo con organizaciones en el Ecuador y b) que tengan presencia constante en redes sociales y medios digitales. De acuerdo con el primer criterio y para garantizar la diversidad, se incluyó solamente a una organización por país europeo y a organizaciones de distintos tamaños. Todas las organizaciones fueron contactadas y dieron su consentimiento previo al inicio del estudio.

A lo largo de 18 meses (01.2014 - 06.2015) el estudio siguió las cuentas de Facebook y Twitter de cinco organizaciones europeas de redes de organizaciones de movimientos sociales y sus ramas locales para el Ecuador: Instituto Humanista para la Cooperación al Desarrollo, Hivos (Países Bajos), IBIS (Dinamarca), Oxfam Intermón (España), Sociedad Sueca para la Conservación de la Naturaleza (SSNC - Suecia) y Vredeseilanden (Veco, Bélgica) ${ }^{4}$.

Históricamente todas las organizaciones estudiadas surgieron como movimientos sociales que posterior- mente se institucionalizaron y se establecieron como organizaciones de la sociedad civil, ampliando su alcance de acción fuera de sus fronteras nacionales para convertirse en "TANs" y luego formar parte de movimientos globales por la justicia social y actualmente, parafraseando a Kavada (2010), su "activismo se ha transformado hacia lo digital".

Cabe hacer tres puntualizaciónes sobre las cuentas estudiadas: 1) IBIS fue incluida ya que hasta fines de 2013 contaba con una oficina regional con trabajo en el Ecuador, la cual fue cerrada en 2014 y por ende no se incluyó a ninguna organización social; 2) Oxfam Intermón pese a tener una oficina nacional en Ecuador, esta no contó con redes sociales, por ello tampoco se la incluyó, y 3) Redmanglar Internacional se incluyó como socio local de la SSNC, esta red reúne a organizaciones latinoamericanas con miembros en el Ecuador.

Toda la información proveniente de Twitter y la mayor parte de la información de Facebook fue recolectada automáticamente mediante el uso de NVivo10 y su extensión NCapture, un programa de análisis de información cualitativa. El interfaz de programación de aplicaciones de Facebook (API, por sus siglas en inglés: application pro-

\footnotetext{
${ }^{4}$ La organización belga Vredeseilanden (islas de paz, por su nombre en holandés) en América Latina se conoce como VECO, el acrónimo que la organización utiliza para referirse a sus oficinas locales (Vredeseilanden Country Offices).
} 
gramming interface) impidió la descarga de contenidos de perfiles personales a partir de febrero 2015, esto afectó a la cuenta de Redmanglar Internacional creada como perfil personal, por lo que la información se obtuvo manualmente de febrero a junio 2015.

\section{MAPEAR LA INTERACCIÓN DIGITAL}

La metáfora del rizoma de Deleuze y Guattari desafía al pensamiento hegemónico del "árbol del conocimiento" compuesto por raíces que fortalecen a un tronco centralizado que luego se ramifica. El rizoma, por el contrario se basa en multiplicidades, es heterogéneo y conectado, compuesto por cantidades de raíces que son a la vez tronco y que se entrecruzan y conectan mediante nódulos, esparciéndose y ocupando nuevos territorios y definiendo dimensiones y no unidades que tienen principio pero no fin. Seis principios lo caracterizan:

- Conexión: cualquier nodo puede conectarse con cualquier otro nodo.

- Heterogeneidad: el rizoma no es uniforme, sino que se basa en un sinnúmero de diferencias.

- Multiplicidad: procesos similares ocurren en varios lugares y tiempos a la vez.

- Ruptura a-significante: el rizoma puede fraccionarse incluso llegar hasta desaparecer, no obstante este se regenerará en otro lugar y tiempo.
- Cartografía: se puede acceder al rizoma desde sus múltiples entradas y conexiones.

- Decalcomanía: cada nueva repetición del rizoma implica una modificación y mejora gracias al contacto con el exterior durante el proceso de transferencia.

\section{Principio de Cartografía}

Deleuze y Guattari sugieren hacer un mapa y no un trazo para estructurar el pensamiento, ya que el mapa está abierto a una multiplicidad de puntos de acceso y vías de conexión, las cuales son siempre descartadas al diseñar un trazo único que pierde la infinidad de posibilidades de acceso.

La Tabla 1, provee información sobre las cuentas de Facebook y Twitter de las organizaciones estudiadas mientras que la Tabla 2 provee información sobre los datos que constituyen el cuerpo de análisis de este artículo. Este es el mapa sobre el cual trabaja esta investigación y su análisis procura mantener abiertas todas las posibilidades oferta- 
das, so pena de perderse en la vastedad y variedad de datos.

En cuanto a la acción conectiva facilitada por las organizaciones para la interacción digital de los participantes en sus redes, la Figura 1 muestra cómo esta se desarrolla ubicando a la organiza- ción en el centro y cuanto más se aleja la línea del eje central, la comunicación se vuelve menos mediada por la organización y más personalizada, ampliando el rango y las posibilidades de expansión de temáticas y apoyo.

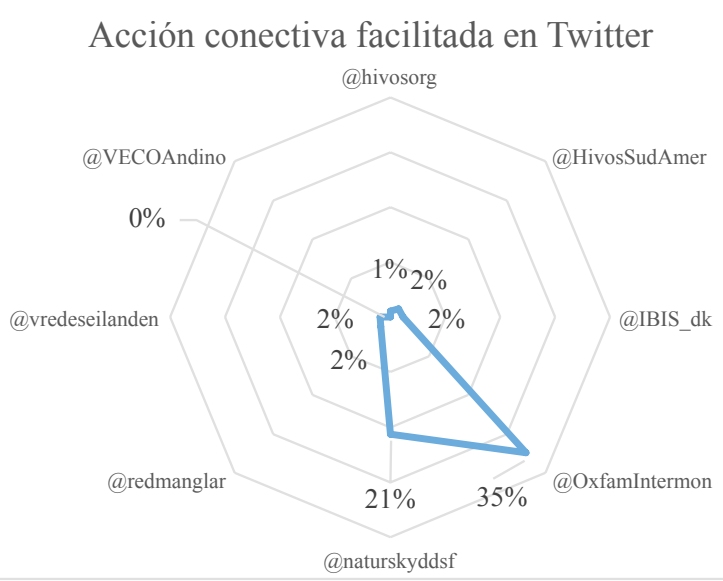

Figura 1: Acción conectiva facilitada en Twitter 
Tabla 1: Actividad en Facebook y Twitter de las organizaciones estudiadas

\begin{tabular}{|c|c|c|c|c|c|}
\hline \multicolumn{6}{|c|}{ a) Facebook ${ }^{5}$} \\
\hline \multicolumn{2}{|c|}{ Organización } & Idioma & Seguidores & Creada & Abierta para publicar \\
\hline \multirow[t]{2}{*}{1} & Hivos (Holanda) & Holandés ${ }^{6}$ & 9,571 & 21.12.2010 & $\sqrt{ }$ \\
\hline & Hivos América del Sur & Español & 2,200 & 30.04 .2013 & \\
\hline 2 & IBIS Denmark & Danés & 11,214 & 29.09 .2009 & $\sqrt{ }$ \\
\hline 3 & Oxfam Intermón & Español & 88,825 & 07.12 .2010 & $\sqrt{ }$ \\
\hline \multirow[t]{2}{*}{4} & Naturskyddsförening (SSNC) & Sueco & 139,017 & 19.01.2010 & $\sqrt{ }$ \\
\hline & Redmanglar Internacional & Español & 2,572 & 20.10.2011 & $\sqrt{ }$ \\
\hline \multirow[t]{2}{*}{5} & Vredeseilanden & Holandés & 6,682 & 15.07.2009 & $\sqrt{ }$ \\
\hline & VECO Andino & Español & 764 & 05.04 .2014 & $\sqrt{ }$ \\
\hline
\end{tabular}

\section{b) Twitter ${ }^{7}$}

\begin{tabular}{|c|l|l|l|c|c|c|}
\hline \multicolumn{2}{|c|}{ Organización } & \multicolumn{1}{c|}{ Cuenta } & Idioma & Seguidores & Siguiendo & Creada \\
\hline \multirow{2}{*}{1} & Hivos International & @hivosorg & Inglés & 2,992 & 1,263 & 02.02 .2014 \\
\cline { 2 - 7 } & Hivos América del Sur & @HivosSudAmer & Español & 641 & 203 & 30.04 .2013 \\
\hline \multirow{2}{*}{2} & IBIS Denmark & @|BIS_dk & Danés & 1270 & 959 & 12.08 .2011 \\
\hline \multirow{2}{*}{3} & Oxfam Intermón & @0xfamIntermon & Español & $48.9 K$ & 1,599 & 23.07 .2009 \\
\hline \multirow{2}{*}{4} & Naturskyddsförening (SSNC) & @naturskyddsf & Sueco & $16.4 K$ & 1,601 & 27.08 .2009 \\
\cline { 2 - 7 } & Redmanglar Internacional & @redmanglar & Español & 185 & 641 & 21.09 .2014 \\
\hline \multirow{2}{*}{5} & Vredeseilanden & @vredeseilanden & Holandés & 1,854 & 1,131 & 21.10 .2007 \\
\cline { 2 - 7 } & VECO Andino & @VECCAndino & Español & 4 & 20 & 31.03 .2015 \\
\hline
\end{tabular}

\footnotetext{
${ }^{5}$ Datos recuperados el 25.09.2015

${ }^{6}$ Aunque el perfil de esta página está en inglés, las publicaciones son mayoritariamente en holandés.

${ }^{7}$ Datos recuperados el 16.09.2015
} 


\begin{tabular}{|c|c|c|c|c|c|c|c|c|c|c|c|c|}
\hline \multirow{3}{*}{ 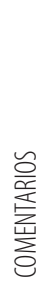 } & $\stackrel{\curvearrowright}{\wedge}$ & b̊ํ. & & த̊ & $\stackrel{\text { શે }}{=}$ & $\stackrel{\stackrel{\circ}{m}}{m}$ & & $\begin{array}{l}80 \\
\text { छ̊ }\end{array}$ & \&̊ & ৪̊ & \&̊ & \\
\hline & $\begin{array}{l}\dot{E} \\
\bar{\delta} \\
\bar{\delta}\end{array}$ & $\begin{array}{l}\stackrel{0}{2} \\
\infty \\
\infty \\
\end{array}$ & & $\begin{array}{l}\text { o̊ } \\
\text { wi } \\
\end{array}$ & $\begin{array}{l}\text { ลे } \\
\text { ஓे }\end{array}$ & 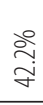 & & 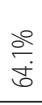 & ฏें & $\begin{array}{l}\stackrel{8}{0} \\
\stackrel{+}{+}\end{array}$ & 六 & \\
\hline & ह் & $\frac{2^{\circ}}{\frac{y}{y}}$ & & 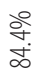 & $\frac{2}{2}$ & $\begin{array}{l}\text { ò } \\
\text { in }\end{array}$ & & $\begin{array}{l}\text { के } \\
\text { ज़ं }\end{array}$ & $\begin{array}{l}\text { ळे } \\
\text { ஓं }\end{array}$ & 守 & $\begin{array}{l}\stackrel{0}{2} \\
\text { مे }\end{array}$ & \\
\hline \multirow{3}{*}{ 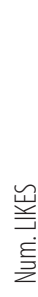 } & $\frac{8}{\wedge}$ & $\begin{array}{l}0 \\
\infty \\
\infty\end{array}$ & & ঐे & $\stackrel{\stackrel{\circ}{n}}{\sim}$ & ڤे & & 离 & i̊̊ & ठ̊ํํ & ठे. & \\
\hline & 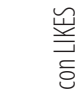 & ஓ̊ํํ & & $\begin{array}{l}\text { वें } \\
\text { वं }\end{array}$ & $\begin{array}{l}\text { ᄋ̊ } \\
\text { ू. }\end{array}$ & $\frac{\stackrel{2}{f}}{\frac{f}{0}}$ & & $\stackrel{\circ}{\stackrel{2}{\circ}}$ & 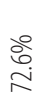 & $\begin{array}{l}\text { ઠे } \\
\text { مू }\end{array}$ & 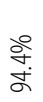 & \\
\hline & 岂 & $\begin{array}{l}\text { ळे } \\
\text { ळे }\end{array}$ & & $\begin{array}{l}\stackrel{8}{\circ} \\
\stackrel{+}{ }\end{array}$ & ठ̊. & 离 & & $\begin{array}{l}80 \\
\text { ঠें }\end{array}$ & 守 & 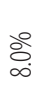 & $\begin{array}{l}\text { bे } \\
\text { ज̊ }\end{array}$ & \\
\hline \multirow{2}{*}{ 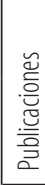 } & 峞 & $\frac{2^{\circ}}{\stackrel{1}{n}}$ & & $\stackrel{\infty}{\Xi}$ & $\begin{array}{l}\stackrel{0}{\delta} \\
\stackrel{+}{ \pm}\end{array}$ & 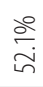 & & $\frac{\stackrel{\circ}{\circ}}{6}$ & $\frac{\stackrel{2}{ٍ}}{\stackrel{n}{m}}$ & 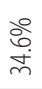 & $\stackrel{\circ}{\check{\pi}}$ & \\
\hline & $\frac{\tilde{\pi}}{\frac{\pi}{0}}$ & 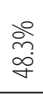 & & $\begin{array}{l}8 \\
8 \\
8\end{array}$ & 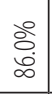 & $\begin{array}{l}\text { ஓे } \\
\text { के }\end{array}$ & & $\stackrel{\substack{n \\
n}}{m}$ & 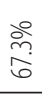 & 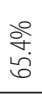 & ふें & \\
\hline \multirow{2}{*}{ 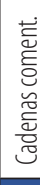 } & $1 \times$ ह் & $\sim n$ & & $\sim$ & $\forall$ & $\mp$ & & $\stackrel{\sim}{\sim}$ & \llcorner & $\sim$ & $\sim$ & \\
\hline & $\frac{\widetilde{\Xi}}{\frac{\pi}{0}}$ & $\stackrel{\infty}{=}$ & & 8 & 志 & $\stackrel{\stackrel{\sim}{N}}{N}$ & & $\stackrel{2}{\approx}$ & $\stackrel{\infty}{\sim}$ & శ్రి & $\bar{\forall}$ & \\
\hline & ह் & $\frac{\infty}{6}$ & & $\stackrel{ }{\sim}$ & हू & $\underset{f}{\tilde{b}}$ & & 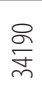 & $\stackrel{\infty}{\Xi}$ & $\bar{\sigma}$ & 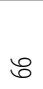 & $\begin{array}{l}\frac{m}{E} \\
\frac{5}{f}\end{array}$ \\
\hline & 这 & $\stackrel{\leftrightarrow}{\Im}$ & & $\stackrel{\Xi}{\Xi}$ & ஜ & 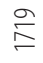 & & ఫ্రి & 员 & $\widetilde{\sigma}$ & $\stackrel{\infty}{\circ}$ & $\begin{array}{l}\Sigma \\
\Sigma \\
\Sigma\end{array}$ \\
\hline 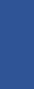 & 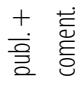 & $\stackrel{\infty}{\varrho}$ & & 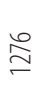 & $\stackrel{o}{o}$ & శ్ర్ & & 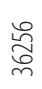 & $\stackrel{\infty}{\stackrel{\curvearrowright}{\sigma}}$ & $\underset{\sim}{\approx}$ & 夺 & 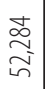 \\
\hline 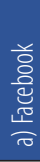 & 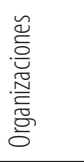 & 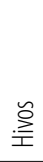 & 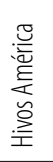 & $\frac{亏}{\frac{\Xi}{8}}$ & $\stackrel{\simeq}{\underline{\underline{O}}}$ & 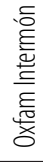 & 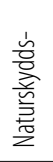 & 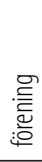 & 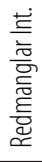 & 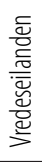 & $\begin{array}{l}\stackrel{ }{\frac{C}{0}} \\
\text { 玄 } \\
\text { 岁 }\end{array}$ & 胥 \\
\hline
\end{tabular}


REVISTA PUCE. ISSN: 2528-8156. NÚM.104.
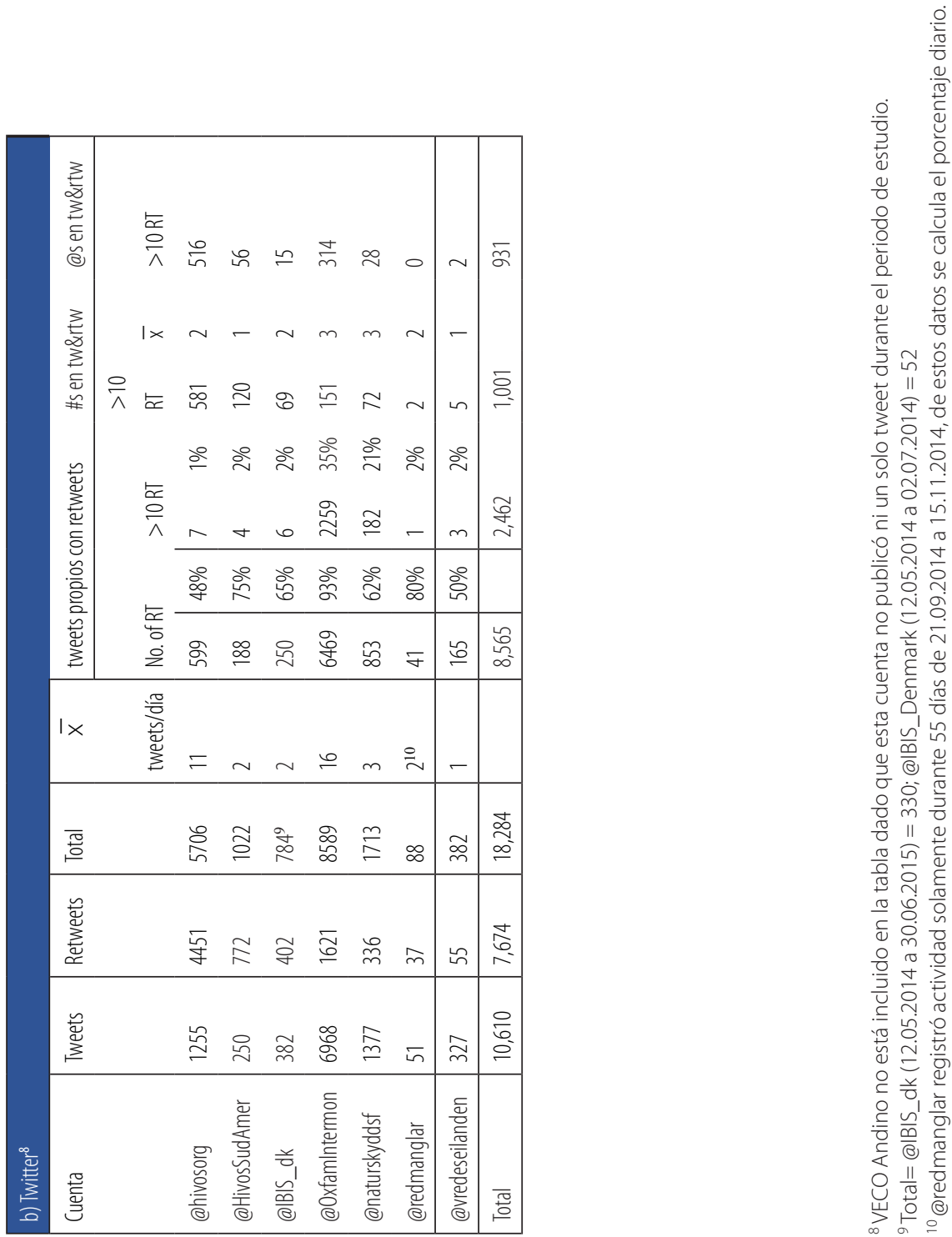
La Figura 2 grafica los datos de la Tabla 2 para mostrar la acción conectiva facilitada en Facebook. Como se observa, Hivos América del Sur no permite que los participantes publiquen en este canal, mientras que la SSNC y Oxfam Intermón muestran un rango mayor de publicación por parte de los participantes que el de la propia organización (67,5\% y $52,1 \%$, respectivamente). Esto no significa que las organizaciones hayan abandonado sus canales de comunicación, sino más bien que estos canales se han abierto como sitios en los que los participantes tienen la oportunidad de reflexionar y debatir libremente.

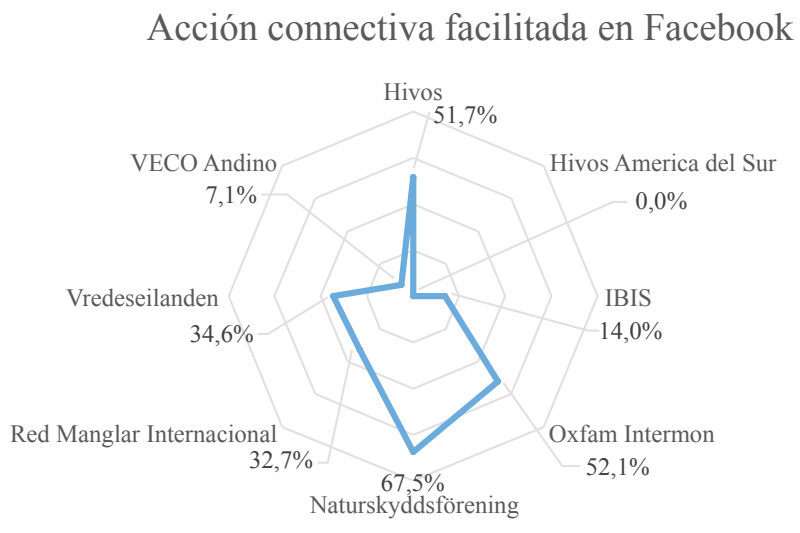

Figura 2: Acción conectiva facilitada en Facebook

El porcentaje de acción conectiva de VECO Andino (7.1\%) e IBIS (14\%) son muy bajos y el de Hivos América del Sur es totalmente ausente (0\%). De acuerdo con la clasificación de Bennetty Segerberg (2013) estas tres organizaciones retratarían a las redes de acción colectiva intermediada por organizaciones que utilizan esta red social como medios para informar y compartir los anhelos de la organización y no para promover la reflexión y la acción.

\section{Principios de Conexión,} Heterogeneidad y Multiplicidad

Los distintos programas, campañas y acciones de las organizaciones estudiadas manejan en muchos casos sus propias redes sociales, que se interconectan a través de publicaciones 
y tweets. Por ejemplo la publicación S_34186"1 de la SSNC se refiere a una publicación compartida procedente de la cuenta de la campaña AntiScampi (en contra del camarón de cultivo):

SSNC: Vi räkbombar restauranger som fortfarande serverar skamräkor. Först ut: Vapiano. Räkbomba du också!

(Estamos bombardeando con camarón a los restaurantes que continúan sirviendo camarón que avergüenza.
El primero: Vapiano. ¡Bombardealo tú también!)

El llamado apunta a que la cadena de restaurantes deje de servir "skamräkor" (camarón que avergüenza), basado en los informes sobre los impactos negativos ambientales y sociales a las comunidades del manglar. Este llamado se dirige a dos audiencias y a dos redes conectadas pero heterogéneas, como se muestra en la Tabla 3.

\section{Tabla 3: Llamado de acción a bombardear camarones}

\begin{tabular}{|l|l|l|l|l|}
\hline $\begin{array}{l}\text { Acción conectiva } \\
\text { bombardear camarón }\end{array}$ & Fecha & Likes & Comentarios & Compartido \\
\hline Antiscampi* & 11.03 .2014 & 2403 & 147 & 749 \\
\hline SSNC** & 14.03 .2014 & 2627 & 64 & -- \\
\hline
\end{tabular}

* Datos recuperados el 01.10 .2015

** Datos recuperados el 20.05.2014

Las dos publicaciones muestran un número similar de Likes y de etiquetas a amigos dentro de los comentarios, una invitación a sus propias redes a que conozcan la acción. Desafortunadamente la publicación no continuó activa en la cuenta de la SSNC durante el análisis, por eso la diferencia de fechas en la recuperación de la información, y los datos archivados no incluyeron información sobre publicaciones compartidas, lo cual es un affordance de Facebook en la actualización del programa de mediados de 2014 que aún no es soportada por NVivo.

El principio de multiplicidad se observa en el contenido del llamado que insta a los participantes a visitar un local de esta cadena (tomar una acción en el mundo físico), solicitar la carta y si se confirma el expendio de camarón de cultivo, abandonar el local dejando una

${ }^{11}$ Esta numeración se refiere a la identificación consecutiva dada por NVivo a los datos recopilados para todas las publicaciones, comentarios y a los comentarios sobre comentarios de Facebook. 
tarjeta en signo de protesta en la mesa. de material de campaña de la organizaEsta tarjeta se puede descargar e impri- ción (véase la Figura 3).

mir desde el link provisto a un repositorio

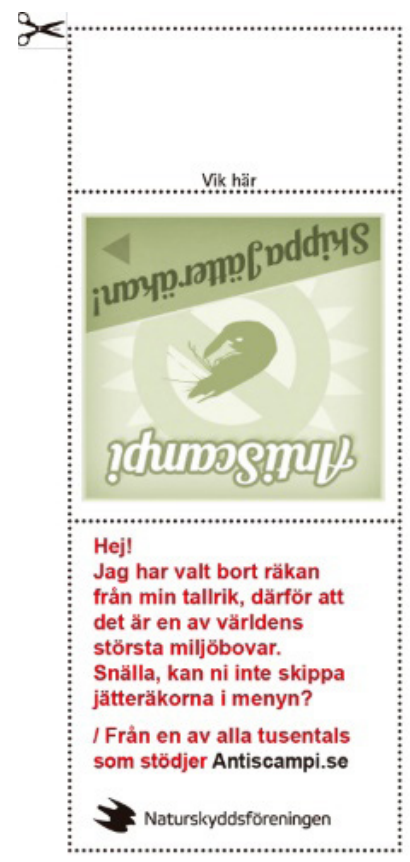

Figura 3: Material para impresión de la campaña AntiScampi 2014²

Los principios de conexión, he- Inicialmente el API de Facebook extraía terogeneidad y multiplicidad también información de los perfiles personales, pueden analizarse en relación a los pero actualmente la única información participantes en la interacción digital. meta accesible es el nombre del usuario

\footnotetext{
${ }^{12}$ La tarjeta dice: "Hola. Yo dejé los camarones fuera de mi plato porque son uno de los más grandes contaminadores a nivel mundial. Por favor, podrían retirar los camarones de cultivo del menú? / De parte de uno de los miles que apoyan Antiscampi.se" Fuente: Beställning av Antiscampi-material 2014. http:// naturskyddsforeningen.wufoo.com/forms/mzem4h71qgeon1/Recuperada el 24.10.2014.
} 
y, de ser provista, su ubicación geográfica. Únicamente el 18\% de las publicaciones de participantes en la cuenta de Oxfam Intermón ofrecieron información para su geolocalización. De todas maneras, esta pequeña muestra despliega un panorama de conexiones desperdigadas alrededor del mundo: principalmente en España, país sede la organización, pero también en África, otras partes de Europa y América del Norte y Sur (véase la Figura 4).

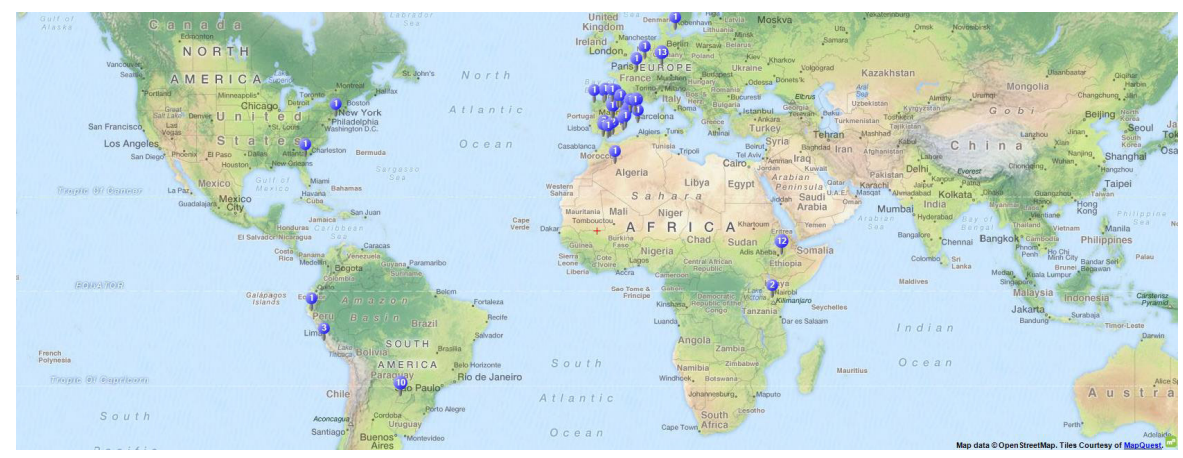

(Mapa generado por NVivo10 13.08.2015)

Figura 4: Geolocalización de las publicaciones en la página de. Oxfam Intermón

Pese a que la comunicación en Twitter no ofrece una conversación encadenada bajo un tweet, esto no significa que no exista interacción digital. Esta puede y debe ser analizada desde las affordances que ofrece Twitter, esto es a través de retweets, hashtags, enlaces y menciones. El estudio buscó a través de ellos interconexiones entre las organizaciones, sin embargo, fueron muy escasas como se muestra en la Tabla 4.

Los retweets y las menciones se dan solamente entre organizaciones socias, así Hivos International reenvía publicaciones de @HivosSudAmer, lo mismo se aplica para IBIS como nuevo miembro de OXFAM. Los retweets de las cuentas de Oxfam no son de su rama española @Oxfamlntermon sino de otras oficinas. Además, IBIS reenvía publicaciones de Hivos sobre la campaña Alliance 2015 en la que ambos son miembros, e Hivos América del Sur envía retweets de la campaña Periodismo de Datos Abiertos de Bolivia, apoyada por Oxfam e Hivos internacional. 
Tabla 4: Retweets y menciones entre organizaciones

\begin{tabular}{|c|c|c|c|c|c|c|c|c|}
\hline Retweets \& menciones & 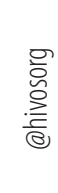 & 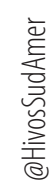 & 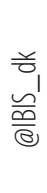 & 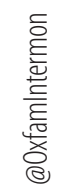 & 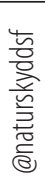 & 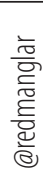 & 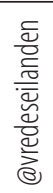 & 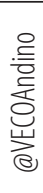 \\
\hline @hivosorg & & 56 & & $15^{*}$ & & & & \\
\hline @HivosSudAmer & 38 & & & $2^{* * *}$ & & & & \\
\hline @|B|S_dk & 1 & & & $25^{* *}$ & & & & \\
\hline \multicolumn{9}{|l|}{ @0xfamlntermon } \\
\hline \multicolumn{9}{|l|}{ @naturskyddsf } \\
\hline \multicolumn{9}{|l|}{ @redmanglar } \\
\hline @vredeseilanden & & & & & & & & \\
\hline
\end{tabular}

*Retweets de 0xfam, oxfamgbpolicy y 0xfamAmerica

**Retweets de 0xfam, oxfaminSA [0xfam en SudAfrica] y 0xfamlndia

***Retweets de 0xfam y oxfam_es

Otro affordance de Twitter para datos analizados tampoco se observa la conexión es el uso compartido de gran conexión en el uso de hashtags, hashtags que crean una red distinta de la Tabla 5 muestra los seis hashtags que aquella conformada por los seguidores/ fueron compartidos por lo menos por siguiendo (Bruns \& Burgess, 2015). En los tres organizaciones.

Tabla 5: Hashtags compartidos por al menos 3 organizaciones

\begin{tabular}{|l|l|l|l|}
\hline$\#$ & No. & Retweets & Tema \\
\hline WorldRefugeeDay & 3 & 922 & 20 de junio, día mundial del refugiado \\
\hline Gaza & 3 & 614 & Conflico 2014 Israel-Gaza \\
\hline NepalEarthquake & 3 & 180 & Abril 2015, terremoto de Nepal \\
\hline inequality & 3 & 636 & Inequidad / Desigualdad \\
\hline bringbackourgirls & 3 & 1389 & 276 niñas secuestradas en Nigeria en abil 2014 \\
\hline COP20 & 3 & 107 & Conferencia 2014 sobe Cambio Climático en el Perú \\
\hline
\end{tabular}

De estos hashtags, el único que \#inequality (desigualdad). Un análisis no se refiere a un evento específico es más apropiado de "hashtags" debe aban- 
donar frecuencia literal de palabras y enfocarse en conceptos claves entrelazados mediante un análisis cualitativo, para lo cual se propone utilizar el principio de decalcomanía en la siguiente sección.

\section{Principio de Decalcomanía}

La interacción digital es dinámica y modelada por sus participantes. Aquí el principio de decalcomanía tiene lugar. Se refiere a la técnica artística de doblar y copiar un mismo dibujo con las consiguientes variaciones resultantes de la transferencia. En el caso de los hashtags el uso de nubes de palabras (word clouds) sirve para visualizar aquellos más relevantes sobre la base de su frecuencia de repetición en cada cuenta por separado. La noción de \#inequality que entrelaza a tres organizaciones, también es enfatizada en la cuenta de Oxfam Intermón a través del hashtag \#IGUALES que utiliza su opuesto afirmativo, otro concepto destacado y relacionado es el de \#STOPDesigualdad, como se visualiza en la Figura 5.

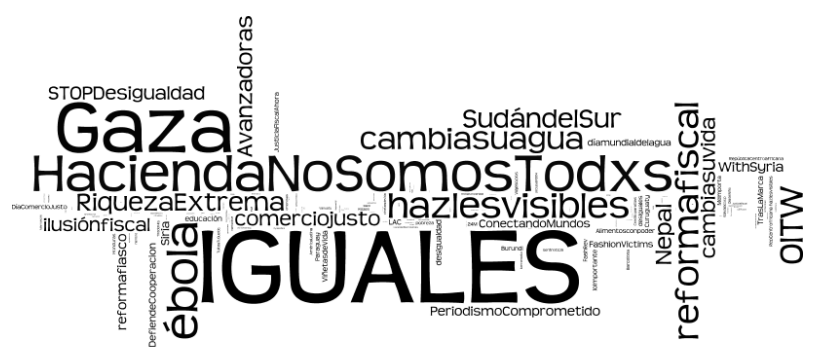

Elaborado con Wordle.net

Figura 5: Retweeted hashtags de 0xfam Intermón

La campaña por la erradicación de la desigualdad económica extrema de Oxfam hizo virales los hashtags \#RiquezaExtrema, \#HaciendaNoSomosTodxs, \#reformafiscal, \#ilusiónfiscal y \#reformafiasco, todos referidos a la reforma fiscal aprobada en España en noviembre de 2014.
Un desafío de este estudio ha sido analizar la interacción digital en los diferentes idiomas de las organizaciones, no obstante en la interacción digital aumentó enormemente el número de otros idiomas empleados por los participantes, como se puede observar en las Tablas 6 y 7. 


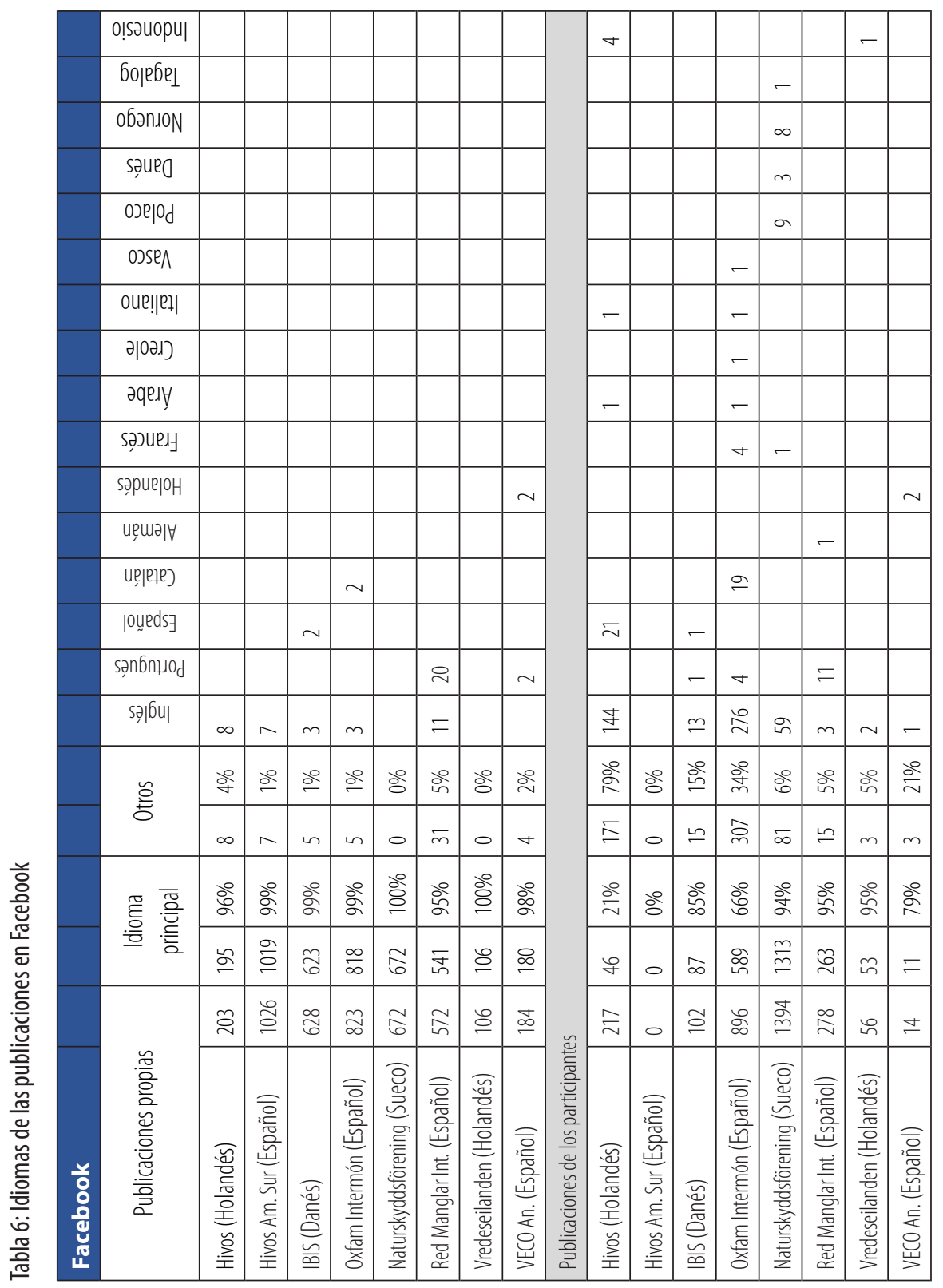




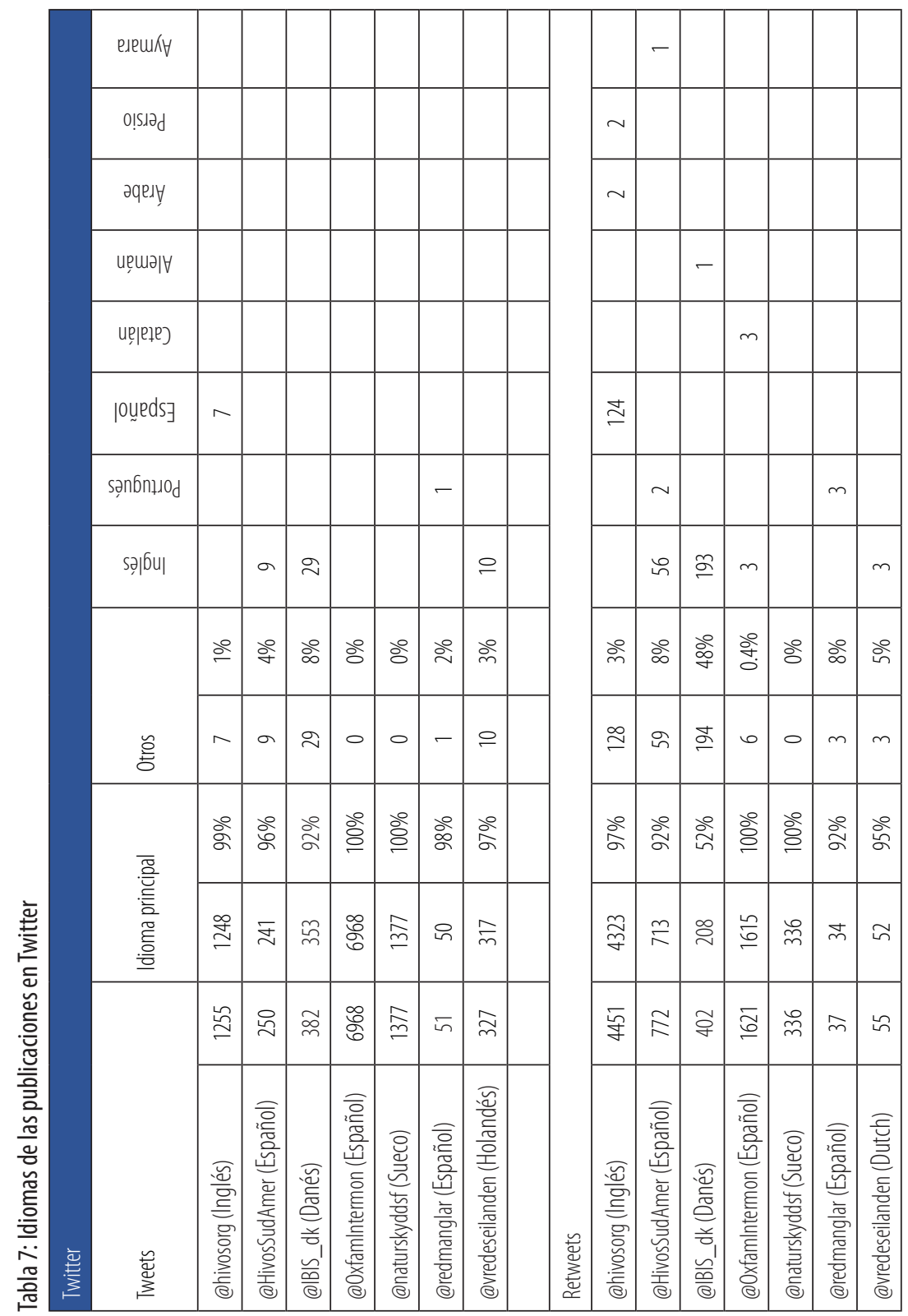


El $99 \%$ de las publicaciones de Oxfam Intermón es en español con tres publicaciones en inglés y dos en catalán. Sin embargo, en la interacción digital solo el $66 \%$ de los participantes publican en español, y el restante $34 \%$ (307 publicaciones) se divide entre 276 en inglés, 4 en portugués, 19 en catalán, 4 en árabe y una publicación en creole, italiano, vasco y polaco.

Las páginas de Facebook facilitan conexiones al publicar en las cuentas de amigos. Así, los participantes abandonan sus propios espacios comunicativos para abrir nuevas línea de conversación en un espacio público facilitado por la organización.

\section{Principio de Ruptura Asignificante}

El ejemplo siguiente se refiere a una pregunta publicada en la cuenta de Facebook de la SSNC:

Till nästa jul undrar jag om det är en bra idé att byta fungerande lampor i adventljusstakar och julgranbelysning till ledlampor. Eller är det resursslöseri och slänga lampor som fungerar?

(Para la próxima Navidad, me pregunto sies una buena idea reemplazar las luces que están funcionando en la corona de adviento y el árbol de Navidad por luces LED. O es solo un desperdicio de recursos, tirar las luces en funcionamiento?)
La SSNC es considerada una voz experta en Suecia en la conservación de la naturaleza, por lo tanto la pregunta es relevante y recibió siete comentarios, el primero de la propia SSNC. Los dos Comentarios siguientes reflexionan sobre experiencias propias en el uso de luces LED; el cuarto es una comparación sobre luces led e incandescentes. Pero, tal como sucede en cualquier otro tipo de conversación sea ésta mediada o no, el tema tomó otro rumbo a partir del próximo comentario:

Part.1 Här får alla något att fundera över på nästa flygning över halva jordklotet.

(Aquí, todos tienen algo para pensar en el próximo viaje alrededor del mundo.)

Part.2 Jag flyger väldigt sällan och har aldrig flugit utanför Europa.

(Yo vuelo frecuentemente pero nunca he salido de Europa.)

Part.3 Jag flyger oxå väldigt sällan, har aldrig varit i Thailand och de poppis ställena "på andra sidan jorden". Och det är alltid bättre att göra något, än att inte göra nåt, det trodde jag var common knowledge.

(Yo también vuelo frecuentemente, pero nunca he estado en Tailandia o en otros lugares de moda "al otro lado del mundo" $Y$ siempre es mejor hacer algo 
que no hacer nada, pensé que eso era de "conocimiento popular").

Esta conversación apunta al principio de ruptura asignificante, al desviar la conversación hacia otros temas ale- jados de la organización y el tema propuesto. No obstante, el comentario de cierre regresa la conversación a la pregunta inicial. El rizoma se visualiza resurgiendo desde otro lugar para volver al tema de origen.

\section{DISCUSIÓN}

La metáfora del rizoma se presenta como una herramienta adecuada para analizar la interacción digital que tiene lugar en las redes sociales de Incidencia 2.0 en donde temas, participantes, actividades y conceptos aparentemente desunidos toman fuerza en la interconexión misma. Tal es el caso del principio de decalcomanía que resulta una "técnica" fehaciente para las affordances de los hashtags en la interacción digital. A través de su habilidad para deterritorializarse, es decir desplazarse de un lugar a otro, y en este caso pasar de un idioma a otro completamente diferente, estos hashtags se reproducen, se traducen y transfieren realzando sus características originales dentro de nuevos espacios.

El análisis de los datos recolectados de Twitter provee información acerca de la manera en que la organización se desempeña y de cómo los hashtags y los tweets se esparcen en el ámbito propio de Twitter, sin embargo no provee información sobre las ideas, reflexiones, deseos y anhelos de los participantes conectados a esta red, más allá de las nociones intermediadas por la propia organización que publica tweets y retweets de temas que a la organización le atañen. Por lo tanto, seguir la comunicación en Twitter solamente a través de las cuentas de las organizaciones se limita al primer tipo de redes descritas por Bennettt y Sergerberg, o regresar a un estado de Incidencia 1.0, que no está diseñado para la participación y por ende no promueve una práxis de reflexión y acción para el cambio social.

En los datos analizados las publicaciones directas y los tweets muestran la presencia de por lo menos 18 idiomas diferentes. Sin embargo, las publicaciones en idiomas distintos del principal solamente promueven conversaciones cortas y sin mayor discusión y construcción, por lo que sería exagerado sugerir la presencia de conversaciones multilingües como una affordance de la Incidencia 2.0. El campo del multilingüismo en 
la interacción digital es un tema extenso que requiere de un análisis más profundo el cual queda planteado para futuras investigaciones.

Este artículo sugiere que la estructura de la interacción digital es rizomática, múltiple, variada, abierta, descomplicada, capaz de reproducirse y regenerarse. Sin embargo, es necesario profundizar en el análisis del contenido que fluye a través de los múltiples, hete- rogéneos, diversos y conexos filamentos, raíces y ramas del rizoma de la Incidencia 2.0. Este contenido involucra la praxis de reflexión-acción que propone el campo de la comunicación para el cambio social, comunicación que se vale de herramientas y canales digitales para genera un accionar distinto, personalizado en cada localidad y que será motivo de estudio posterior. 


\section{BIBLIOGRAFIA}

Ackland, R., \& O'Neil, M. (2013). Online collective identity: The case of the environmental movement. In C. Hine (Ed.), Virtual methods: issues in social research on the Internet (Vol. 3, pp. 239-272). London: Sage.

Bennett, W. L., \& Segerberg, A. (2013). The logic of connective action : digital media and the personalization of contentious politics. Cambridge: Cambridge University Press.

Bruns, A., \& Burgess, J. (2015). Twitter Hashtags from Ad Hoc to Calculated Publics. In N. Rambukkana (Ed.), Hashtag publics: the power and politics of discursive networks (pp. 13-28). New York: Peter Lang.

Castells, M. (2012). Redes de indignación y esperanza : los movimientos sociales en la era de internet. Madrid: Alianza Editorial.

Christensen, C. (2011). Twitter Revolutions? Addressing Social Media and Dissent. The Communication Review, 14(3), 155-157. doi:10.108 0/10714421.2011.597235

Conole, G. (2013). Affordances Designing for Learning in an Open World (Vol. 4, pp. 85-100): Springer New York.

Cordoba, D., \& Jansen, K. (2015). Realigning the Political and the Technical: NGOs and the Politicization of
Agrarian Development in Bolivia. European Journal of Development Research, advance online publication 19 March 2015. doi:10.1057

Deleuze, G., \& Guattari, F. (1987). A Thousand Plateaus (B. Massumi, Trans.). Minneapolis: University of Minnesota Press.

Della Porta, D. (2009a). Democracy in social movements. Basingstoke: Palgrave Macmillan.

Della Porta, D. (2009b). Global Justice Movement Organizations: The Organizational Population. In D. Della Porta (Ed.), Democracy in Social Movements (pp. 16-43). Basingstoke: Palgrave Macmillan Ltd.

Della Porta, D., \& Mosca, L. (2005). Global-net for Global Movements? A Network of Networks for a Movement of Movements. Journal of Public Policy, 25(01), 165-190.

Dohn, N. B. (2009). Affordances revisited: Articulating a Merleau-Pontian view. International Journal of Computer-Supported Collaborative Learning, 4(2), 151170. doi:10.1007/s11412-0099062-z

Dohn, N. B., \& Johnsen, L. (2009). E-læring på web 2.0. Frederiksberg: Samfundslitteratur. 
Earl, J., \& Kimport, K. (2011). Digitally enabled social change: activism in the Internet age. Cambridge, Mass: MIT Press.

Escobar, A., \& Osterweil, M. (2010). Social Movements and the Politics of the Virtual: Deleuzian Strategies. In C. B. Jensen \& K. Rødje (Eds.), Deleuzian intersections: science, technology, anthropology (pp. 187217). New York: Berghahn Books.

Flyvbjerg, B. (2006). Five Misunderstandings About Case-Study Research. Qualitative Inquiry, 12(2), 219-245. doi:10.1177/1077800405284363

Freire, P. (1985). Pedagogía del oprimido (35.ed. ed.). Madrid: Siglo veintiuno.

Funke, P. N. (2012). The Global Social Forum Rhizome: A Theoretical Framework. Globalizations, 9(3), 351-364. doi:10.1080/14747731.2 012.680727

Garcia-Alabacete, G., \& Theocharis, Y. (2014). Opportunities and Challenges of Analysing Twitter Content: A Comparison of the Occupation Movements in Spain, Greece and the United States. In M. Cantijoch, R. Gibson, \& S. Ward (Eds.), Analysing social media data and web networks (pp. 119-153). New York, NY; Houndmills, Basingstoke, Hampshire: Palgrave Macmillan.
Kavada, A. (2010). Activism Transforms Digital: The Social Movement Perspective. In M. Joyce (Ed.), Digital Activism Decoded: The New Mechanics of Change (pp. 101118). New York \& Amsterdam: International debate education association.

Kavada, A. (2012). Engagement, bonding, and identity across multiple platforms: Avaaz on Facebook, YouTube, and MySpace. Medie Kultur. Journal of media and communication research, 28(52).

Lauridsen, E., \& Hansen, J. (2016). IPads' affordances i undervisningen. In N. B. Dohn \& J. J. Hansen (Eds.), Didaktik, design og digitalisering (Vol. 9, pp. 153-174): Samfundslitteratur.

Marinucci, M. (2010). You Can't Front on Facebook. In D. E. Wittkower (Ed.), Facebook and philosophy: what's on your mind? (pp. 65-74). Chicago: Open Court.

Nos Aldás, E., \& Pinazo, D. (2013). Communication and Engagement for Social Justice. Peace Review, 25(3), 343-348. doi:10.1080/10402659.2 013.816552

O'Reilly, T. (2007). What Is Web 2.0: Design Patterns and Business Models for the Next Generation of Software. International Journal of Digital Economics, 65, 17-37. 
Shirky, C. (2008). Here comes everybody: the power of organizing without organizations. New York: Penguin Books.

Steger, M. B., Goodman, J., \& Wilson, E. K. (2013). Justice globalism: ideology, crises, policy. London, CA, New Delhi: Sage.

Toret, J. (2013). Tecnopolítica: la potencia de las multitudes conectadas. Retrieved from Universitat Oberta de Catalunya:

Tufte, T. (2013). Towards a Renaissance in Communication for Social Change. In T. Tufte, N. Wildermuth, A. S. Hansen-Skovmoes, \& W. Mitullah (Eds.), speaking up and talking back?: media, empowerment and civic engagement among East and Southern African youth (Vol. 2012/2013). Göteborg: Nordicom.

Tufte, T. (2015). Comunicación para el cambio social. Spain: Icaria editorial.

Wilkins, K. G. (2014). Emerging Issues in Strategic Communication for De- velopment and Social Change. In K. G. Wilkins, T. Tufte, \& R. Obregón (Eds.), The handbook of development communication and social change (pp. 139-144). Chichester: John Wiley \& Sons.

Willetts, P. (2011). Non-governmental organizations in world politics: the construction of global governance (Vol. 49). New York: Routledge.

Yépez-Reyes, V., \& Dohn, N. B. (2016). Affordances for læring i web 2.0: En rodvækstanalyse af kommunikation i organiserede sociale bevægelse. In N. B. Dohn \& J. J. Hansen (Eds.), Didaktik, design og digitalisering (Vol. 9, pp. 175-196): Samfundslitteratur.

Yin, R. K. (2014). Case study research: design and methods (5th ed.). London: Sage Publications.

Zárate, M. d. C. (2015). Resistencias y movimientos sociales transnacionales. Alteridades, 25(Julio-Diciembre), 65-77. 
